\title{
Does exercise test modality influence dyspnoea perception in obese patients with COPD?
}

\author{
Casey E. Ciavaglia', Jordan A. Guenette ${ }^{1,2}$, Josuel Ora ${ }^{1,3}$, Katherine A. Webb ${ }^{1}$, \\ J. Alberto Neder ${ }^{1}$ and Denis E. O'Donnell ${ }^{1}$
}

Affiliations: 'Respiratory Investigation Unit, Dept of Medicine, Queen's University and Kingston General Hospital, Kingston, ON, and '2Dept of Physical Therapy and UBC Centre for Heart Lung Innovation, University of British Columbia, Vancouver, BC, Canada. ${ }^{3}$ Dept of Internal Medicine, “Tor Vergata" Polyclinic Hospital, Rome, Italy.

Correspondence: D.E. O’Donnell, 102 Stuart Street, Kingston, ON, K7L 2V6, Canada.

E-mail: odonnellaqueensu.ca

ABSTRACT The purpose of this study was to investigate whether differences in physiological responses to weight-bearing (walking) and weight-supported (cycle) exercise influence dyspnoea perception in obese chronic obstructive pulmonary disease (COPD) patients, where such discrepancies are probably exaggerated.

We compared metabolic, ventilatory and perceptual responses during incremental treadmill and cycle exercise using a matched linearised rise in work rate in 18 (10 males and eight females) obese (mean \pm SD body mass index $36.4 \pm 5.0 \mathrm{~kg} \cdot \mathrm{m}^{-2}$ ) patients with COPD (forced expiratory volume in $1 \mathrm{~s} 60 \pm 11 \%$ predicted).

Compared with cycle testing, treadmill testing was associated with a significantly higher oxygen uptake, lower ventilatory equivalent for oxygen and greater oxyhaemoglobin desaturation at a given work rate $(\mathrm{p}<0.01)$. Cycle testing was associated with a higher respiratory exchange ratio $(\mathrm{p}<0.01)$, earlier ventilatory threshold $(\mathrm{p}<0.01)$ and greater peak leg discomfort ratings $(\mathrm{p}=0.01)$. Ventilation, breathing pattern and operating lung volumes were similar between tests, as were dyspnoea/work rate and dyspnoea/ventilation relationships.

Despite significant between-test differences in physiological responses, ventilation, operating lung volumes and dyspnoea intensity were similar at any given external power output during incremental walking and cycling exercise in obese COPD patients. These data provide evidence that either exercise modality can be selected for reliable evaluation of exertional dyspnoea in this population in research and clinical settings.

@ERSpublications

Reliable evaluations of exertional dyspnoea can be obtained by cycle or treadmill exercise tests in obese COPD patients http://ow.ly/tQZdU

This article has supplementary material available from www.erj.ersjournals.com

Received: Aug 302013 | Accepted after revision: Nov 172013 | First published online: Dec 052013

Support statement: This study was supported by funds from the Ontario Thoracic Society grant-in-aid 2009/2010 competition. J.A. Guenette was supported by post-doctoral fellowships from the Natural Sciences and Engineering Research Council of Canada, the Canadian Thoracic Society and the Canadian Lung Association, and a New Investigator Award from the Providence Health Care Research Institute and St Paul's Hospital Foundation.

Conflict of interest: Disclosures can be found alongside the online version of this article at www.erj.ersjournals.com

Copyright (CERS 2014 


\section{Introduction}

The prevalence of both obesity and chronic obstructive pulmonary disease (COPD) is increasing steadily throughout the world [1]. The combination of these common conditions is associated with increased activity restriction and healthcare burden [2]. The effective management of exercise intolerance in obese COPD patients remains a major challenge and awaits a better understanding of the underlying mechanisms.

It is widely believed that obese COPD patients experience greater dyspnoea during daily activities than normal-weight COPD patients. However, while field tests indicate reduced walking distance in obese versus normal-weight COPD patients [3,4], physiological studies using cycle ergometry have found that dyspnoea intensity, endurance time and peak oxygen uptake $\left(V^{\prime} \mathrm{O}_{2}\right)$ are similar in the two groups when severity of airway obstruction is matched $[5,6]$. The question therefore arises: does measurement of perceived dyspnoea intensity and exercise performance in obese COPD during cycle ergometry (where leg muscles are selectively stressed $[7,8]$ ) accurately reflect the situation during daily activities such as walking? In this regard, no studies have compared the relationship between dyspnoea and physiological responses during carefully matched walking and cycling exercise protocols in obese patients with COPD.

It has previously been demonstrated that the dynamic respiratory mechanical derangements, and the associated dyspnoea, seen during exercise in COPD rise in direct proportion to the prevailing ventilatory requirement of the task [9]. It follows that differences in dyspnoea intensity between cycling and walking could occur at a given power output if the metabolic and ventilatory responses to these tasks are different. In the obese COPD patient, the relative importance of the higher metabolic cost of external work during treadmill exercise or the earlier metabolic acidosis of cycle exercise in driving ventilation (and consequent dyspnoea intensity) is difficult to predict [10-12]. It is also conceivable that differences in dynamic respiratory mechanics or in the source of the ventilatory stimulation (i.e. metabolic loading, skeletal muscle recruitment, arterial oxygen saturation and haemodynamic responses) between the two exercise modalities could influence the dyspnoea/ventilation relationship.

Accordingly, the main objective of this study was to better understand the influence of the physiological peculiarities of each exercise modality on dyspnoea perception in patients with combined obesity and COPD. We compared physiological responses, dyspnoea/work rate and dyspnoea/ventilation relationships during symptom-limited incremental cycle and treadmill exercise tests, using matched linearised work rate protocols.

\section{Methods}

Subjects

18 clinically stable, obese (body mass index $(\mathrm{BMI})>30 \mathrm{~kg} \cdot \mathrm{m}^{-2}$ ) COPD patients (forced expiratory volume in $1 \mathrm{~s}(\mathrm{FEV} 1) /$ forced vital capacity $<70 \%)$ aged $40-80$ years with a post-bronchodilator $\mathrm{FEV} 1<80 \%$ predicted were recruited. Patients were excluded if they had significant disease that affected breathlessness or exercise capacity (i.e. metabolic, cardiovascular, neuromuscular and/or musculoskeletal conditions), received daytime oxygen therapy, had significant respiratory disease other than COPD, were too breathless to leave the house, were too fit (Medical Research Council dyspnoea scale 5 or 1 , respectively) or had any contraindications to clinical exercise testing.

\section{Study design}

This cross-sectional study received approval from the Queen's University health sciences and affiliated teaching hospitals research ethics board (DMED-1187-09). After obtaining informed consent, subjects attended three visits, each separated by $\geqslant 48 \mathrm{~h}$. At visit 1 , all subjects underwent medical screening, detailed pulmonary function tests, and familiarisation with all exercise testing procedures. Visits 2 and 3 included pulmonary function tests and either a cycle or treadmill test (randomised visit order). Before each visit, subjects withheld short-acting $\beta_{2}$-agonist and anticholinergic bronchodilators for $\geqslant 4$ and $6 \mathrm{~h}$, respectively, and long-acting bronchodilators for $\geqslant 12 \mathrm{~h}$. Subjects were instructed to avoid caffeine, heavy meals, alcohol and major physical exertion prior to each visit. Subjects underwent a dual-energy X-ray absorptiometry (DEXA) scan within the study period to quantify body composition. When available, prior clinical chest computed tomography (CT) scan results were used to provide qualitative assessments of emphysema.

\section{Procedures}

Pulmonary function testing included routine spirometry, body plethysmography, single-breath diffusing capacity of the lung for carbon monoxide (DLCO) and maximum voluntary ventilation (MVV) using automated testing equipment (Vs62j body plethysmograph with Vmax229d; SensorMedics, Yorba Linda, CA, USA). Cardiopulmonary exercise tests were performed on an electronically braked cycle ergometer (Ergometrics 800S; SensorMedics) and on a treadmill (Medtrack ST55; Quinton Instrument Company, 
Bothell, WA, USA) using a Vmax229d Cardiopulmonary Exercise Testing System (SensorMedics). Cycle and treadmill exercise tests were both performed using 10-W increments, which increased every 2 min to a symptom-limited end-point. The incremental treadmill protocol was individualised based on body weight: there was a linear increase in speed and a curvilinear rise in grade (online supplementary material). Subjects rated "breathing discomfort" and "leg discomfort" on a modified 10-point Borg scale [13]. Inspiratory capacity manoeuvres were performed during a steady-state resting period, during each stage of exercise and at peak exercise. Operating lung volumes were derived from inspiratory capacity measurements, as previously described [14]. Immediately after exercise, subjects were asked why they stopped exercising. Breath-by-breath data from the last $30 \mathrm{~s}$ of loaded pedalling were averaged for each individual and analysed as "peak" exercise. Three independent observers, who were blinded to exercise modality, identified the ventilatory threshold ( $\left.V^{\prime} \mathrm{Th}\right)$ using the "V-slope" plot, which was then double-checked with the inflection points suggested by the ventilatory equivalent and end-tidal pressure methods [15].

\section{Statistical analysis}

A sample size of 18 provided $80 \%$ power to detect a minimal clinically important difference in dyspnoea of \pm 1 Borg scale unit measured at a standardised work rate [16], assuming $\alpha=0.05$ and a within-patient SD of 1 unit. Comparisons of exercise modalities were made at rest, at standardised work rates (i.e. 10, 20, 30 and $40 \mathrm{~W}$ ) and at peak exercise using paired two-tailed t-tests. Data are presented as mean \pm SD.

\section{Results}

\section{Subjects}

Subject characteristics and DEXA scan results are presented in tables 1 and 2, respectively. Subjects had moderate airflow obstruction and lung volumes were within the predicted normal ranges with the exception of residual volume (124\% pred) and expiratory reserve volume (66\% pred). Evidence of emphysema was shown on all available chest CT scans (13 out of 18 subjects). DEXA scans from 15 subjects (equipment was unavailable for the remaining three subjects) revealed that total body mass was elevated by $15.5 \mathrm{~kg}(17.5 \%)$ in males and $16.1 \mathrm{~kg}(21.7 \%)$ in females compared to the population means for age-matched individuals

\section{TABLE 1 Subject characteristics}

\begin{tabular}{|c|c|}
\hline Age years & $66 \pm 8$ \\
\hline Male/female $n$ & $10 / 8$ \\
\hline Height $\mathrm{cm}$ & $167.2 \pm 2.0$ \\
\hline Weight kg & $102.1 \pm 20.1$ \\
\hline Ideal body weight kg & $69.3 \pm 2.0$ \\
\hline Body mass index $\mathrm{kg} \cdot \mathrm{m}^{-2}$ & $36.4 \pm 5.0$ \\
\hline \multicolumn{2}{|l|}{ Waist circumference $\mathrm{cm}$} \\
\hline Male & $129 \pm 10$ \\
\hline Female & $115 \pm 7$ \\
\hline Smoking history pack-years & $50 \pm 32$ \\
\hline COPD duration years & $8.8 \pm 4.4$ \\
\hline Baseline dyspnoea index 0-12 & $6.6 \pm 1.8$ \\
\hline MRC dyspnoea scale $0-5$ & $2.4 \pm 0.7$ \\
\hline \multicolumn{2}{|l|}{ Pulmonary function } \\
\hline FEV1 L (\% predicted) & $1.40 \pm 0.40(60 \pm 11)$ \\
\hline FVC L (\% predicted) & $3.08 \pm 0.82(88 \pm 14)$ \\
\hline $\mathrm{FEV}_{1} / \mathrm{FVC} \%$ & $48 \pm 9$ \\
\hline IC L (\% predicted) & $2.41 \pm 0.76(90 \pm 23)$ \\
\hline FRC L & $3.31 \pm 0.71(105 \pm 19)$ \\
\hline RV L & $2.67 \pm 0.68(124 \pm 33)$ \\
\hline TLC L & $5.73 \pm 1.07(98 \pm 13)$ \\
\hline RV/TLC \% & $47 \pm 8$ \\
\hline sRaw \% pred & $385 \pm 151$ \\
\hline DLCO $\mathrm{mL} \cdot \mathrm{mmHg}^{-1} \cdot \mathrm{min}^{-1}$ (\% predicted) & $12.2 \pm 4 . \overline{4}(50 \pm 12)$ \\
\hline DLCo/VA \% pred & $76 \pm 16$ \\
\hline
\end{tabular}

Data are presented as mean $\pm S D$, unless otherwise stated. COPD: chronic obstructive pulmonary disease; MRC: Medical Research Council; FEV1: forced expiratory volume in $1 \mathrm{~s}$; FVC: forced vital capacity; IC: inspiratory capacity; FRC: functional residual capacity; RV: residual volume; TLC: total lung capacity; sRaw: specific airway resistance; DLCO: diffusing capacity of the lung for carbon monoxide; VA: alveolar volume. 
[17]; this resulted from a greater total fat mass (males $11.0 \mathrm{~kg}$ and females $7.1 \mathrm{~kg}$ ) and lean mass (males $4.5 \mathrm{~kg}$ and females $8.8 \mathrm{~kg}$ ), compared to the population means.

\section{Responses to cycle and treadmill exercise}

Subjects reached a similar peak work rate during cycle and exercise tests (table 3). Although exercise duration was longer by a mean difference of $52 \mathrm{~s}$ in the treadmill versus cycle test, this was not statistically significant $(\mathrm{p}=0.11)$. The distribution of the main reasons for stopping exercise did not differ between tests $(\mathrm{p}=0.56)$ : breathing discomfort (treadmill $\mathrm{n}=10$ and cycle $\mathrm{n}=8)$, leg discomfort (treadmill $\mathrm{n}=2$ and cycle $n=5$ ) and a combination of breathing and leg discomfort (treadmill $n=6$ and cycle $n=5$ ). Rest and peak exercise data are presented in table 3.

\section{Metabolic and gas exchange responses}

Peak $\mathrm{V}^{\prime} \mathrm{O}_{2}$ expressed as \% pred was higher during treadmill compared with cycle testing $(91 \pm 30 \%$ pred versus $78 \pm 26 \%$ pred, respectively; $\mathrm{p}<0.05$ ) using the recommended sex-specific predictive equations for obese males [18] and obese females [19] described by LORENZO and BABB [20]. There was a significant upward displacement of $\mathrm{V} \mathrm{O}_{2}$ relative to work rate during treadmill exercise compared with cycle exercise. The $V \mathrm{O}_{2}$-work rate slope was $14.2 \pm 2.1 \mathrm{~mL} \cdot \mathrm{min}^{-1} \cdot \mathrm{W}^{-1}$ and $12.5 \pm 3.7 \mathrm{~mL} \cdot \mathrm{min}^{-1} \cdot \mathrm{W}^{-1}$ during treadmill exercise and cycle exercise, respectively $(\mathrm{p}=0.07)$ (fig. 1$)$. Carbon dioxide production $\left(\mathrm{VCO}_{2}\right)$ was higher at $30 \mathrm{~W}$ and $40 \mathrm{~W}$ and at the peak of treadmill exercise compared with cycling (fig. 1). The respiratory exchange ratio (RER) during cycle tests compared with treadmill tests showed an upward shift from the onset of exercise that was maintained through to peak exercise (fig. 1). 16 subjects had an identifiable $V^{\prime}$ Th. Subjects reached an earlier $V^{\prime} \mathrm{Th}$ during cycle exercise, at a $V^{\prime} \mathrm{O}_{2}$ of $1.01 \pm 0.23 \mathrm{~L} \cdot \mathrm{min}^{-1}$ compared with $1.17 \pm 0.27 \mathrm{~L} \cdot \mathrm{min}^{-1}$ during treadmill exercise $(\mathrm{p}<0.05)$. $V^{\prime}$ Th relative to predicted peak $V \mathrm{O}_{2}$ was similar between modalities and above the lower limit of normal (cycle 53\% and treadmill 58\%) [18]. Subjects experienced greater arterial oxygen desaturation during treadmill exercise compared with cycle exercise, with peak decreases of $-5.2 \pm 3.2 \%$ and $-3.2 \pm 2.2 \%$, respectively $(\mathrm{p}=0.01)$ (fig. 1$)$.

\section{Cardiac responses}

Heart rate rose in a similar fashion between modalities throughout submaximal exercise, but reached $\sim 9$ beats $\cdot \mathrm{min}^{-1}$ higher $(\mathrm{p}<0.05)$ at peak treadmill exercise compared with peak cycle exercise (table 3$)$.

\section{Ventilatory responses}

Minute ventilation $\left(V^{\prime} \mathrm{E}\right)$ was not different between test modalities at any given work rate (fig. 1). $V^{\prime} \mathrm{E} / V^{\prime} \mathrm{O}_{2}$ was greater at all stages of cycle exercise compared with treadmill exercise (fig. 1), but $V^{\prime} \mathrm{E} / V^{\prime} \mathrm{CO}_{2}$ was only greater at $40 \mathrm{~W}$ and at the peak of cycle exercise (table 3), and $V \mathrm{E} / V^{2} \mathrm{CO}_{2}$ slopes were similar (fig. 2). Breathing pattern and operating lung volumes were not statistically different at a given work rate or $V^{\prime} \mathrm{E}$ between modalities throughout exercise (fig. 2). Subjects reached critical respiratory mechanical reserves at

TABLE 2 Dual-energy $X$-ray absorptiometry scan results

\begin{tabular}{|c|c|c|c|c|c|}
\hline & \multirow{2}{*}{$\begin{array}{l}\text { All obese COPD } \\
\text { subjects }\end{array}$} & \multicolumn{2}{|c|}{ Male } & \multicolumn{2}{|c|}{ Female } \\
\hline & & Obese COPD & Population average ${ }^{\#}$ & Obese COPD & Population average \\
\hline Subjects & 15 & 8 & & 7 & \\
\hline \multicolumn{6}{|l|}{ Total body } \\
\hline Mass kg & $97.7 \pm 12.2$ & $104.2 \pm 11.0$ & 88.7 & $90.2 \pm 9.1$ & 74.1 \\
\hline Lean mass $\mathrm{kg}$ & $56.0 \pm 8.1$ & $62.3 \pm 5.4$ & 57.8 & $48.9 \pm 3.1$ & 40.1 \\
\hline Fat mass $\%$ total body mass & $40.2 \pm 4.9$ & $37.5 \pm 4.4$ & 31.2 & $43.2 \pm 4.0$ & 42.5 \\
\hline \multicolumn{6}{|l|}{ Trunk } \\
\hline Mass kg & $51.3 \pm 8.1$ & $55.8 \pm 7.6$ & 45.8 & $46.2 \pm 5.3$ & 37.1 \\
\hline Fat $\%$ total trunk mass & $42.4 \pm 5.1$ & $41.1 \pm 5.8$ & 33.1 & $43.5 \pm 4.6$ & 41.2 \\
\hline \multicolumn{6}{|l|}{ Both legs } \\
\hline Mass kg & $29.9 \pm 3.6$ & $30.3 \pm 3.3$ & 27.0 & $29.3 \pm 4.0$ & 24.5 \\
\hline Lean mass kg & $17.4 \pm 2.5$ & $19.1 \pm 2.9$ & 17.7 & $15.4 \pm 1.2$ & 12.3 \\
\hline Fat mass $\mathrm{kg}$ & $11.5 \pm 2.9$ & $10.2 \pm 1.9$ & 8.1 & $13.1 \pm 3.3$ & 11.3 \\
\hline Fat $\%$ total leg mass & $38.5 \pm 6.9$ & $33.5 \pm 4.1$ & 29.7 & $44.3 \pm 4.6$ & 45.5 \\
\hline
\end{tabular}

Data are presented as $n$ or mean \pm SD, unless otherwise stated. COPD: chronic obstructive pulmonary disease. " : population averages are means for non-Hispanic white people aged 60-79 years. Data from [17]. 
TABLE 3 Measurements during symptom-limited incremental cycle and treadmill exercise

\begin{tabular}{|c|c|c|c|c|}
\hline & \multicolumn{2}{|c|}{ Rest } & \multicolumn{2}{|c|}{ Peak } \\
\hline & Cycle & Treadmill & Cycle & Treadmill \\
\hline Work rate $\mathrm{W}$ & $0 \pm 0$ & $0 \pm 0$ & $73 \pm 20$ & $78 \pm 28$ \\
\hline Exercise time min:s & $0 \pm 0$ & $0 \pm 0$ & $14: 13 \pm 01: 15$ & $15: 05 \pm 01: 06$ \\
\hline Dyspnoea Borg scale & $0.1 \pm 0.2$ & $0.1 \pm 0.2$ & $6.6 \pm 2.8$ & $6.6 \pm 2.4$ \\
\hline Leg discomfort Borg scale & $0.1 \pm 0.3$ & $0.2 \pm 0.3$ & $6.6 \pm 2.9$ & $5.0 \pm 2.7 * *$ \\
\hline $\mathrm{VO}_{2} \mathrm{~L} \cdot \mathrm{min}^{-1}$ & $0.41 \pm 0.14$ & $0.42 \pm 0.14$ & $1.53 \pm 0.40$ & $1.79 \pm 0.52$ \\
\hline $\mathrm{VO}_{2} \%$ pred maximum & $20.4 \pm 6.7$ & $20.5 \pm 4.9$ & $78.1 \pm 25.7$ & $90.7 \pm 30.3^{* *}$ \\
\hline$V^{\prime} \mathrm{CO}_{2} \mathrm{~L} \cdot \mathrm{min}^{-1}$ & $0.35 \pm 0.12$ & $0.34 \pm 0.12$ & $1.49 \pm 0.39$ & $1.65 \pm 0.51^{* *}$ \\
\hline RER & $0.84 \pm 0.06$ & $0.82 \pm 0.09$ & $0.98 \pm 0.07$ & $0.92 \pm 0.10 * *$ \\
\hline$V E L \cdot \min ^{-1}$ & $14.8 \pm 4.2$ & $15.1 \pm 4.2$ & $47.4 \pm 10.8$ & $49.3 \pm 13.7$ \\
\hline$V T \mathbf{L}$ & $0.78 \pm 0.21$ & $0.85 \pm 0.35$ & $1.30 \pm 0.34$ & $1.34 \pm 0.41$ \\
\hline$f_{R}$ breaths $\cdot \min ^{-1}$ & $20 \pm 5.3$ & $20 \pm 6.0$ & $36 \pm 6.1$ & $37 \pm 7.1$ \\
\hline tו/tтот & $0.38 \pm 0.05$ & $0.38 \pm 0.04$ & $0.40 \pm 0.04$ & $0.41 \pm 0.04$ \\
\hline IC L & $2.50 \pm 0.75$ & $2.44 \pm 0.71$ & $1.84 \pm 0.37$ & $1.79 \pm 0.47$ \\
\hline VT/IC \% & $32.5 \pm 9.9$ & $34.6 \pm 8.5$ & $72.3 \pm 11.1$ & $74.6 \pm 9.2$ \\
\hline IRV L & $1.73 \pm 0.69$ & $1.59 \pm 0.48$ & $0.51 \pm 0.22$ & $0.45 \pm 0.18$ \\
\hline EELV L & $3.31 \pm 0.79$ & $3.4 \pm 0.79$ & $3.97 \pm 0.92$ & $4.07 \pm 0.94$ \\
\hline EILV L & $4.09 \pm 0.82$ & $4.30 \pm 0.89$ & $5.31 \pm 1.12$ & $5.42 \pm 1.13^{*}$ \\
\hline EILV/TLC \% & $70.7 \pm 7.9$ & $72.8 \pm 6.1$ & $91.1 \pm 3.8$ & $92.1 \pm 3.5^{*}$ \\
\hline VE/MVV \% & $29 \pm 1$ & $29 \pm 1$ & $90 \pm 2$ & $92 \pm 2$ \\
\hline$V \mathrm{E} / \mathrm{VO}_{2}$ & $36.8 \pm 5.0$ & $37.1 \pm 7.7$ & $31.7 \pm 6.1$ & $28.0 \pm 5.9 * *$ \\
\hline$V \mathrm{E} / V \mathrm{CO}_{2}$ & $43.9 \pm 6.8$ & $45.3 \pm 7.7$ & $32.4 \pm 5.0$ & $30.4 \pm 4.8^{* *}$ \\
\hline $\mathrm{PETCO}_{2} \mathrm{mmHg}$ & $34 \pm 2.8$ & $33 \pm 3.8$ & $37 \pm 5.5$ & $39 \pm 6.0^{* *}$ \\
\hline Heart rate beats $\cdot \min ^{-1}$ & $77 \pm 2.7$ & $79 \pm 10.2$ & $119 \pm 12.8$ & $128 \pm 14.6 * *$ \\
\hline $\mathrm{SpO}_{2} \%$ & $95 \pm 1.8$ & $95 \pm 2.0$ & $92 \pm 2.7$ & $90 \pm 3.3^{* *}$ \\
\hline
\end{tabular}

Data are presented as mean $\pm \mathrm{SD} . \mathrm{VO}_{2}$ : oxygen uptake; $\mathrm{V}^{\prime} \mathrm{CO}_{2}$ : carbon dioxide production; RER: respiratory exchange ratio; $V E$ : minute ventilation; $V T$ : tidal volume; $f R$ : respiratory frequency; $t / / t T O T$ : inspiratory duty cycle; IC: inspiratory capacity; IRV: inspiratory reserve volume; EELV: end-expiratory lung volume; EILV: endinspiratory lung volume; TLC: total lung capacity; VE/MVV: ventilatory equivalent for maximum voluntary ventilation; $V \mathrm{E} / \mathrm{VO}_{2}$ : ventilatory equivalent for oxygen uptake; $V \mathrm{E} / \mathrm{VCO}_{2}$ : ventilatory equivalent for carbon dioxide production; $\mathrm{PETCO}_{2}$ : end-tidal carbon dioxide tension; $\mathrm{SpO}_{2}$ : arterial oxygen saturation measured by pulse oximetry. ${ }^{*}: p<0.05 ;{ }^{* *}: p<0.01$ cycle versus treadmill.

an end-inspiratory lung volume (EILV) $>90 \%$ of total lung capacity (TLC) and an inspiratory reserve volume (IRV) of $\sim 0.5 \mathrm{~L}$ during both exercise modalities at peak exercise (table 3 ).

\section{Perceptual responses}

Dyspnoea intensity ratings at standardised work rates and at peak exercise were similar between modalities (fig. 3). Leg discomfort was also similar during exercise up to $40 \mathrm{~W}$, but became significantly $(\mathrm{p}<0.05)$ greater at the peak of cycle exercise compared with treadmill exercise (fig. 3). Relationships between dyspnoea intensity and both $V E$ and IRV were not different during cycle exercise and treadmill exercise (fig. 3).

\section{Discussion}

The main findings of the study are as follows. 1) Compared with cycle testing, treadmill testing was associated with a higher $V^{\prime} \mathrm{O}_{2}$, a lower $V^{n} \mathrm{E} / V^{\prime} \mathrm{O}_{2}$ ratio and a greater oxyhaemoglobin desaturation for a given work rate. 2) Cycle testing was associated with a higher RER, an earlier $V^{\prime} T$ and a greater peak leg discomfort rating. 3) Ventilation, breathing pattern, operating lung volumes and dyspnoea intensity were similar at comparative work rates. 4) Despite physiological differences, exercise modality had no effect on the dyspnoea/work rate or dyspnoea/ $V$ E relationships in obese patients with COPD.

Participants had mild to severe obesity with body weights in excess of ideal body weight by an average of $32.8 \mathrm{~kg}$. DEXA scans confirmed increased adipose mass deposition compared to the average for agematched individuals. The largely preserved inspiratory capacity and functional residual capacity probably reflects the known effects of increasing BMI on lung volume components in COPD [3, 21]. Participants had moderate airway obstruction, but, surprisingly, average DLCO was diminished to $50 \%$ pred (possibly reflecting underlying emphysema) in the absence of significant lung hyperinflation. Patients reported 

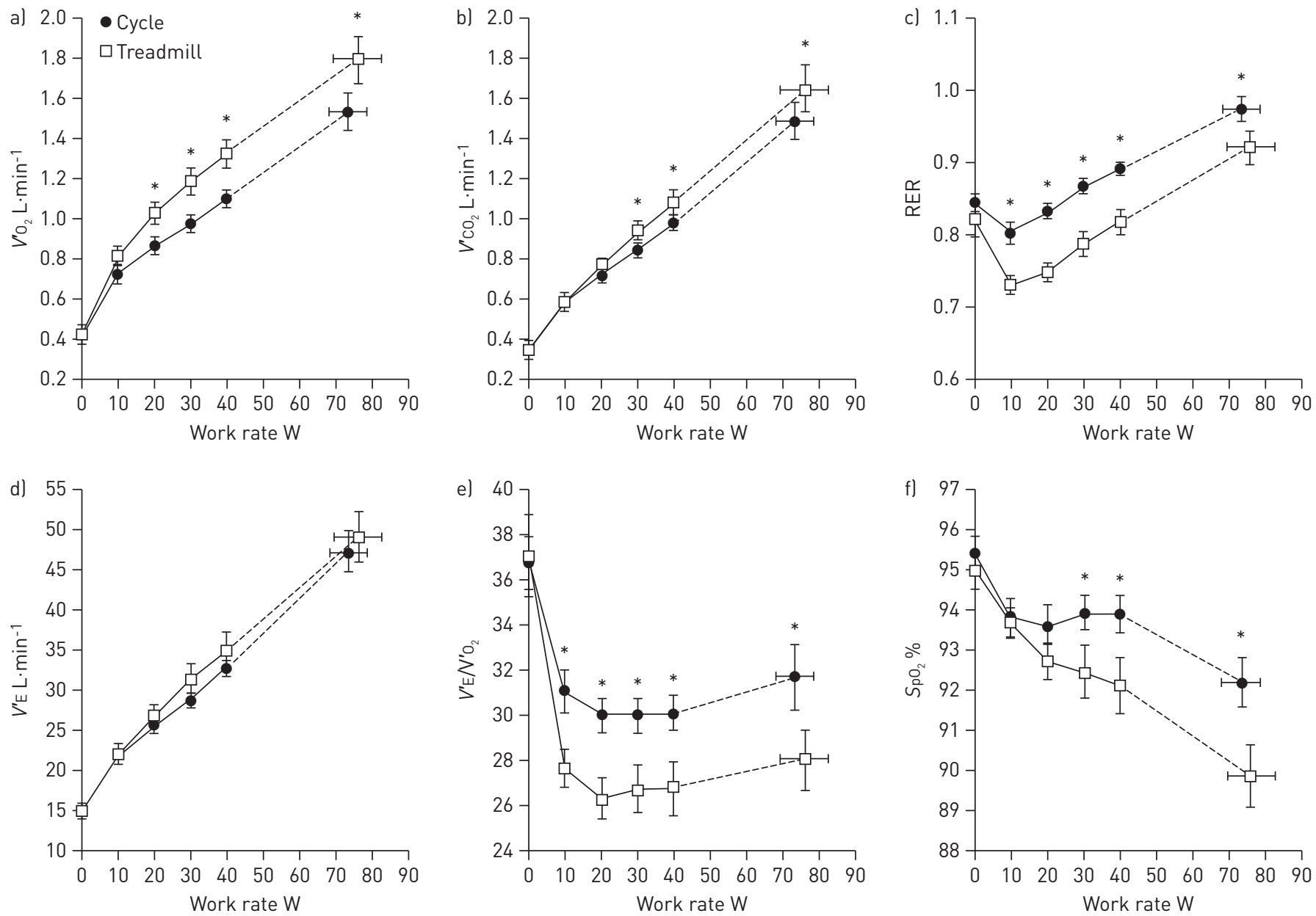

FIGURE 1 a) Oxygen consumption $\left(V^{\circ} \mathrm{O}_{2}\right)$, b) carbon dioxide production $\left(V^{\prime} \mathrm{CO}_{2}\right)$, c) respiratory exchange ratio (RER), d) minute ventilation $\left(V^{\mathrm{f}} \mathrm{E}\right)$, e) ventilatory equivalent for oxygen consumption $\left(\mathrm{V}^{\mathrm{E}} / \mathrm{V}^{\mathrm{O}} \mathrm{O}_{2}\right)$, and $\mathrm{f}$ ) arterial oxygen saturation $\left(\mathrm{SpO}_{2}\right)$ are shown relative to work rate during cycle exercise compared with treadmill exercise. Data are presented as mean \pm SEM. ${ }^{*}: \mathrm{p}<0.05$ cycle versus treadmill at a standardised work rate or at peak exercise.

moderate chronic activity-related dyspnoea but had reasonably preserved cardiorespiratory fitness as assessed by peak $\mathrm{VO}_{2}$ relative to the predicted value based on ideal body weight.

The incremental exercise protocols were well matched for rate of increase in work rate and were of sufficient duration for accurate assessment of perceptual and physiological responses. Peak $\mathrm{V}^{2} \mathrm{O}_{2}$ was significantly higher (by 17\%) during treadmill exercise compared with cycle exercise (fig. 1), probably reflecting the larger skeletal muscle mass recruitment previously described in health [22]. Of note, patients had significant mechanical constraints on ventilation as evidenced by a low breathing reserve (high $V \mathrm{E} / \mathrm{MVV}$ ) and a high EILV/TLC ratio at relatively low peak work rates of $73 \mathrm{~W}$ and $78 \mathrm{~W}$ during cycle and treadmill exercise, respectively. In contrast, participants had adequate cardiac reserve at peak exercise, reaching a mean heart rate of $72 \%$ and $77 \%$ of the predicted maximum during cycle and treadmill tests, respectively.

\section{The dyspnoea/work rate relationship}

A key finding of the present study was the similarity in dyspnoea/work rate relations during both exercise modalities. This indicates that differences in the metabolic cost of external work, $V^{\prime} \mathrm{E} / V^{\prime} \mathrm{O}_{2}, \mathrm{RER}, V^{\prime} \mathrm{Th}$, oxyhaemoglobin saturation, perceived leg discomfort and cardio-circulatory responses did not influence dyspnoea perception at a given power output during cycling and walking in obese COPD patients. The preserved dyspnoea/work rate relationship ultimately reflected the between-test similarity in $V \mathrm{E}$, breathing pattern and operating lung volumes when the increase in work rate was carefully matched. It is remarkable that, despite the differences in potential sources of ventilatory drive, $V \mathrm{E}$ remained tightly coupled with "pulmonary" $V \mathrm{CO}_{2}$ regardless of the exercise modality. These findings are in line with those from on- and off-exercise kinetics studies where the time course of $V^{\prime} \mathrm{E}$ has been found to closely follow the $V^{\prime} \mathrm{CO}_{2}$, and not $V^{\prime} \mathrm{O}_{2}$ or arterial partial pressure of oxygen [23]. 

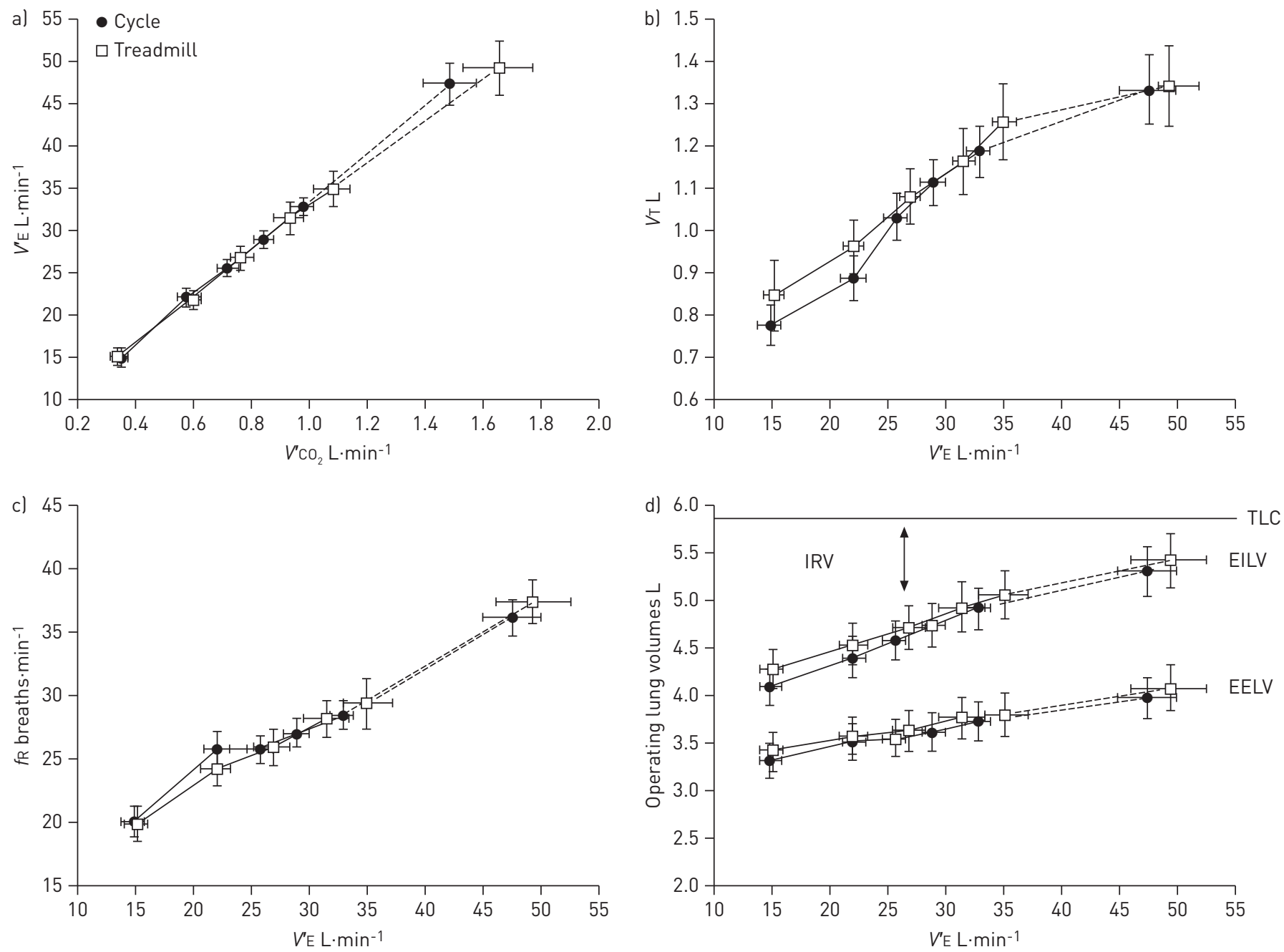

FIGURE 2 a) The relationship between minute ventilation $\left(V^{\prime} \mathrm{E}\right)$ and carbon dioxide production $\left(V^{\prime} \mathrm{CO}_{2}\right)$ was similar during cycle exercise compared with treadmill exercise, b) tidal volume $(V \mathrm{~T}), \mathrm{c})$ respiratory frequency $(f \mathrm{R})$ and d) operating lung volumes expressed relative to $V^{\prime} \mathrm{E}$ were also similar during cycle and treadmill exercise. IRV: inspiratory reserve volume; TLC: total lung capacity; EILV: end-inspiratory lung volume; EELV: end-expiratory lung volume. Data are presented as mean \pm SEM.

\section{The dyspnoea/ventilation relationship}

Our results show that the effective coupling of ventilation to the metabolic demand of comparable external work, expressed as $V^{\prime} \mathrm{CO}_{2}$, is achieved by different mechanisms in cycling and treadmill tests. A higher RER $\left(V \mathrm{CO}_{2} / V^{\prime} \mathrm{O}_{2}\right.$ ) during cycling versus walking (or running) has been previously described in healthy individuals and may reflect preferential carbohydrate utilisation during cycling exercise [24]. We have previously shown that metabolic pathways in leg muscle are altered in COPD such that there is increased dependence on glycolysis and blood glucose utilisation (rather than free fatty acids) compared with healthy individuals [25]. The current study could not determine the extent to which the higher RER during cycling in our obese COPD patients can be explained by preferential utilisation of carbohydrate by the contracting leg muscles. Regardless of the mechanism(s), these muscle metabolic differences between cycling and walking are likely to have contributed to higher $V^{2} \mathrm{CO}_{2}$ (and $V^{2} \mathrm{E}$ ) for a given $V \mathrm{O}_{2}$ in the former modality.

The smaller locomotor muscle mass required to generate the same power output during cycling as walking means that the average metabolic rate per unit of contracting muscle mass is greater with cycling, which can force earlier metabolic acidosis [26]. Our findings of an earlier $V^{\top} \mathrm{Th}$, increased $V^{\mathrm{E}} / V^{\prime} \mathrm{O}_{2}$, preserved oxyhaemoglobin saturation (at a given $V \mathrm{O}_{2}$ ) and reduced peak end-tidal carbon dioxide tension during cycling are consistent with the results of recent studies which, in addition, show relatively increased alveolar ventilation during cycling [10]. It remains possible (but unproven) that differential ventilatory stimulation by mechanoreceptor/metaboreceptor activation (type III and IV afferents) between active locomotor muscle groups (peculiar to the exercise test modality) may explain differences in $V \mathrm{E} / \mathrm{V}^{\mathrm{O}} \mathrm{O}_{2}$ [27]. 

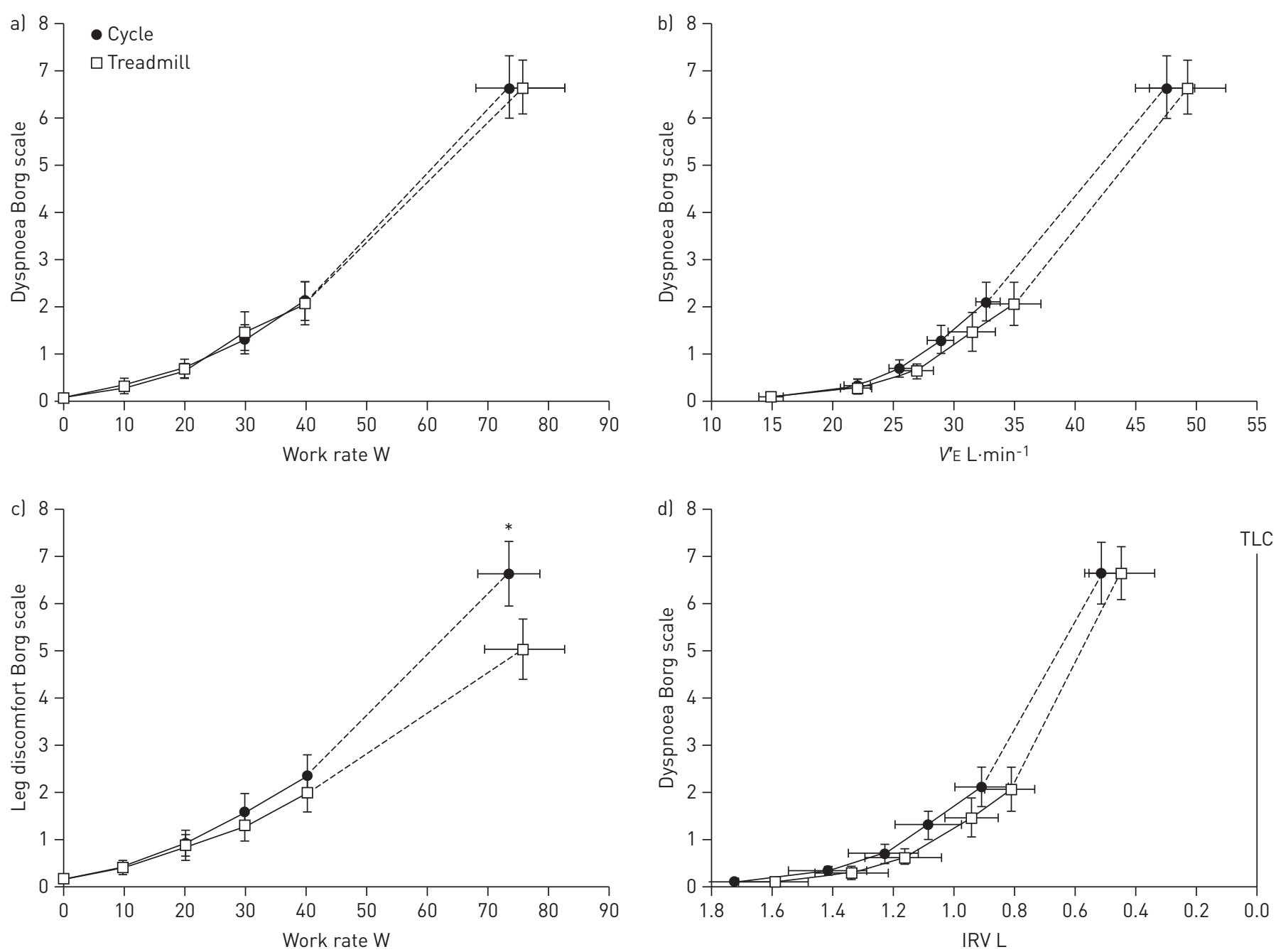

FIGURE 3 Relationships between dyspnoea intensity and a) work rate, b) minute ventilation ( $\left.V^{\mathrm{E}} \mathrm{E}\right)$ and d) inspiratory reserve volume (IRV) were similar during cycle exercise compared to treadmill exercise. c) Intensity of leg discomfort relative to work rate was similar up to $40 \mathrm{~W}$, then became significantly greater by peak exercise during cycle exercise compared with treadmill exercise. Data are presented as mean \pm SEM. TLC: total lung capacity. ${ }^{*}: \mathrm{p}<0.05$.

Despite the previously outlined differences in the sources of ventilatory stimulation during the two exercise modalities, perceived dyspnoea intensity was similar at comparable $V^{\prime} \mathrm{E}$ in obese COPD patients. Based on previous studies, the dyspnoea/ $V$ E relationship during exercise in COPD is altered by change in dynamic respiratory mechanics but not by experimental manipulation of the central respiratory controller per se $[28,29]$. Thus, the preservation of the dyspnoea/ $V^{\prime} \mathrm{E}$ relationship in the current study mainly reflects the similarities in operating lung volumes at a given $V^{\prime} \mathrm{E}$ during cycling and walking, regardless of differences in metabolic loading and acid-base balance.

\section{Limitations}

Since dynamic respiratory mechanics and the extent of metabolic loading differ in normal-weight COPD patients from those in obese patients matched for airflow obstruction $[3,30]$, the lack of effect of exercise modality on dyspnoea intensity seen in this study cannot be generalised to non-obese patients. The present study was performed in patients with moderate COPD and our results may not be generalised to patients with more severe disease. However, we believe that given the close link of work to ventilation, dyspnoea/ work rate and dyspnoea/ventilation relationships are likely to be similar across disease severity if the work performed on different test modalities is closely matched, as in the current study. Measurements of blood lactate, arterial and mixed venous blood gases would be required to better elucidate the observed betweentest differences in RER and oxyhaemoglobin saturation, but this was not the main focus of the current study. 


\section{Conclusions/implications}

Despite consistent task-specific differences in physiological derangements that can influence the central respiratory controller, perception of dyspnoea intensity was most closely linked to carbon dioxide output and the attendant ventilatory and dynamic respiratory mechanical response. Dyspnoea/work rate and dyspnoea/ $V E$ relationships were independent of exercise modality in obese COPD patients. Our results provide reassurance that either exercise modality can be selected for the reliable evaluation of dyspnoea in obese COPD patients, both in research and clinical settings. In obese COPD patients, the higher peak $V^{\prime} \mathrm{O}_{2}$ and lower oxyhaemoglobin saturation during treadmill exercise (compared with cycle exercise) have potentially important implications for the individualised evaluation of cardiorespiratory fitness and pulmonary gas exchange abnormalities, respectively. Another important clinical implication of the results is that interventions that reduce carbon dioxide output (exercise training and energy substrate manipulation) during physical activity in obese COPD patients should effectively relieve exertional dyspnoea.

\section{Acknowledgements}

The authors wish to thank D. Langer and A. Elbehairy (Respiratory Investigation Unit, Dept of Medicine, Queen's University and Kingston General Hospital, Kingston, ON, Canada) for their assistance in determining ventilatory thresholds during exercise and L. Yang (Respiratory Investigation Unit, Dept of Medicine, Queen's University and Kingston General Hospital) for her assistance with patient testing.

\section{References}

1 Finucane MM, Stevens GA, Cowan MJ, et al. National, regional, and global trends in body-mass index since 1980: systematic analysis of health examination surveys and epidemiological studies with 960 country-years and 9.1 million participants. Lancet 2011; 377: 557-567.

2 Vozoris NT, O'Donnell DE. Prevalence, risk factors, activity limitation and health care utilization of an obese population-based sample with chronic obstructive pulmonary disease. Can Respir J 2012; 19: 18-24.

3 Sava F, Laviolette L, Bernard S, et al. The impact of obesity on walking and cycling performance and response to pulmonary rehabilitation in COPD. BMC Pulm Med 2010; 10: 55.

4 Bautista J, Ehsan M, Normandin E, et al. Physiologic responses during the six minute walk test in obese and nonobese COPD patients. Respir Med 2011; 105: 1189-1194.

5 Ora J, Laveneziana P, Ofir D, et al. Combined effects of obesity and chronic obstructive pulmonary disease on dyspnea and exercise tolerance. Am J Respir Crit Care Med 2009; 180: 964-971.

6 Laviolette L, Sava F, O’Donnell DE, et al. Effect of obesity on constant workrate exercise in hyperinflated men with COPD. BMC Pulm Med 2010; 10: 33.

7 Pepin V, Saey D, Whittom F, et al. Walking versus cycling: sensitivity to bronchodilation in chronic obstructive pulmonary disease. Am J Respir Crit Care Med 2005; 172: 1517-1522.

8 Man WD, Soliman MGG, Gearing J, et al. Symptoms and quadriceps fatigability after walking and cycling in chronic obstructive pulmonary disease. Am J Respir Crit Care Med 2003; 168: 562-567.

9 O'Donnell DE, Laveneziana P. Dyspnea and activity limitation in COPD: mechanical factors. COPD 2007; 4: 225-236.

10 Mahler DA, Gifford AH, Waterman LA, et al. Mechanism of greater oxygen desaturation during walking compared with cycling in patients with COPD. Chest 2011; 140: 351-358.

11 Palange $\mathrm{P}$, Forte S, Onorati $\mathrm{P}$, et al. Ventilatory and metabolic adaptations to walking and cycling in patients with COPD. J Appl Physiol 2000; 88: 1715-1720.

12 Hsia D, Casaburi R, Pradhan A, et al. Physiological responses to linear treadmill and cycle ergometer exercise in COPD. Eur Respir J 2009; 34: 605-615.

13 Borg GA. Psychophysical bases of perceived exertion. Med Sci Sports Exerc 1982; 14: 377-381.

14 O'Donnell DE, Revill SM, Webb KA. Dynamic hyperinflation and exercise intolerance in chronic obstructive pulmonary disease. Am J Respir Crit Care Med 2001; 164: 770-777.

15 Gaskill SE, Ruby BC, Walker AJ, et al. Validity and reliability of combining three methods to determine ventilatory threshold. Med Sci Sports Exerc 2001; 33: 1841-1848.

16 Ries AL. Minimally clinically important difference for the UCSD Shortness of Breath Questionnaire, Borg scale, and visual analog scale. COPD 2005; 2: 105-110.

17 Borrud LG, Flegal KM, Looker AC, et al. Body composition data for individuals 8 years of age and older: U.S. population, 1999-2004. Vital Health Stat 2010; 11: 1-87.

18 Wasserman K, Hansen JE, Sue DY, et al. Normal values. In: Principles of exercise testing and interpretation: including pathophysiology and clinical applications. Philadelphia, Lippincott Williams and Wilkins, 2005; pp. $160-182$.

19 Riddle W, Younes M, Remmers JE, et al. Graphical analysis of patient performance in the pulmonary function laboratory. Proc Annu Symp Comput Appl Med Care 1980; 1: 282-290.

20 Lorenzo S, Babb TG. Quantification of cardiorespiratory fitness in healthy nonobese and obese men and women. Chest 2012; 141: 1031-1039.

21 O’Donnell DE, Deesomchok A, Lam YM, et al. Effects of BMI on static lung volumes in patients with airway obstruction. Chest 2011; 140: 461-468.

22 Shephard RJ. Tests of maximum oxygen intake. A critical review. Sports Med 1984; 1: 99-124.

23 Whipp BJ, Ward SA, Lamarra N, et al. Parameters of ventilatory and gas exchange dynamics during exercise. J Appl Physiol Respir Environ Exerc Physiol 1982; 52: 1506-1513.

24 Chenevière X, Malatesta D, Gojanovic B, et al. Differences in whole-body fat oxidation kinetics between cycling and running. Eur J Appl Physiol 2010; 109: 1037-1045.

25 Green HJ, Bombardier E, Burnett M, et al. Organization of metabolic pathways in vastus lateralis of patients with chronic obstructive pulmonary disease. Am J Physiol Regul Integr Comp Physiol 2008; R935-R941. 
26 Koyal SN, Whipp BJ, Huntsman D, et al. Ventilatory responses to the metabolic acidosis of treadmill and cycle ergometry. J Appl Physiol 1976; 40: 864-867.

27 Gagnon P, Bussières JS, Ribeiro F, et al. Influences of spinal anesthesia on exercise tolerance in patients with chronic obstructive pulmonary disease. Am J Respir Crit Care Med 2012; 186: 606-615.

28 Peters MM, Webb KA, O'Donnell DE. Combined physiological effects of bronchodilators and hyperoxia on exertional dyspnoea in normoxic COPD. Thorax 2006; 61: 559-567.

29 Jensen D, Alsuhail A, Viola R, et al. Inhaled fentanyl citrate improves exercise endurance during high-intensity constant work rate cycle exercise in chronic obstructive pulmonary disease. J Pain Symptom Manage 2012; 43: 706-719.

30 Ora J, Laveneziana P, Wadell K, et al. Effect of obesity on respiratory mechanics during rest and exercise in COPD. J Appl Physiol 2011; 111: 10-19. 